\title{
Cuarta Revolución Industrial: gobierno y buena gobernanza
}

Revista Latinoamericana de Economía y Sociedad Digital

Issue 2, agosto 2021

Autores: Alejandro Pisanty Baruch(D) Ernesto Velasco Sánchez(D)

DOI: $10.53857 /$ ALFT6819

Publicado: 25 agosto, 2021

Recibido: 17 marzo, 2021

Cita sugerida: Pisanty Baruch, Alejandro \& Velasco Sánchez, Ernesto (2021) "Cuarta Revolución Industrial, gobierno y buena gobernanza", en Revista Latinoamericana de Economía y Sociedad Digital, Issue 2

Licencia: Creative Commons Atribución-NoComercial 4.0 Internacional (CC BY-NC 4.0)

Tipo: Ensayo

Palabras clave: administración pública, gobernanza multiactor, gobierno, internet de las cosas, multistakeholder, tecnologías

\section{Resumen}

La Cuarta Revolución Industrial o su cuasi-sinónimo Tecnología 4.0 es un constructo útil para analizar una etapa de desarrollo, en curso, de las tecnologías avanzadas y su relación con la sociedad. Presentamos una breve descripción de los retos para los países de América Latina en su aplicación en la Administración Pública y en su gobernanza. El gobierno puede establecer muchos tipos de relaciones con estas tecnologías 4.0: puede ser un promotor activo de la innovación o ser más un espectador, con múltiples otras opciones intermedias. Igualmente, puede ser un promotor del aprovechamiento de los avances tecnológicos para replantearse en alguna medida la relación con la sociedad, abriéndose retos de transparencia y rendición de cuentas; y puede ser proactivo o reticente a usar estas tecnologías para mejorar la prestación de servicio y reorganizarse internamente, con los riesgos políticos y éticos de una burocracia automatizada. Todas estas facetas implican cambio de tal velocidad y granularidad, que los esquemas tradicionales de mando y control no parecen adecuados para enfrentar los retos que presenta la CRI. Proponemos explorar y construir un sistema de gobernanza multisectorial. 


\section{Abstract}

The Fourth Industrial Revolution or its quasi-synonymous Technology 4.0 is a useful construct to analyze a stage of ongoing development of the advanced technologies and its relationship with the society. We present a brief description of the challenges for the LatinAmerican countries in its application in the Public Administration and its governance. The government can establish different types of relationships with these technologies 4.0: they can actively promote innovation or be a spectator with multiple intermediate options. At the same time, they can promote the technological advances to reconsider the relationship with the society to some extent, presenting challenges for transparencies and rendering of accounts and they can be proactive or reluctant to use these technologies to improve the provision of services and reorganize internally, with the political and ethical risks of automated bureaucracy. All these facets imply a change of such speed and granularity that the traditional schemes of command and control do not seem adequate to face the challenges that the Fourth Industrial Revolution CRI presents. We propose to explore and build a multisectorial governance system.

\section{Resumo}

A Quarta Revolução Industrial ou seu quase sinônimo Tecnologia 4.0 é uma construção útil para analisar uma etapa de desenvolvimento, em andamento, de tecnologias avançadas e sua relação com a sociedade. Apresentamos uma breve descrição dos desafios para os países da América Latina no que se refere à sua aplicação na Administração Pública e em sua governança. O governo pode estabelecer muitos tipos de relações com essas tecnologias 4.0: pode ser um promotor ativo de inovação ou ser mais um observador, com diversas outras opções intermediárias. Da mesma forma, pode promover o aproveitamento dos avanços tecnológicos para repensar de certa forma a relação com a sociedade, abrindo-se a desafios de transparência e prestação de contas; e pode ser pró-ativo ou relutante em usar essas tecnologias para melhorar a prestação de serviços e se reorganizar internamente, com os riscos políticos e éticos de uma burocracia automatizada. Todas essas facetas implicam mudanças de tamanha velocidade e detalhamento que os esquemas tradicionais de comando e controle não parecem adequados para enfrentar os desafios apresentados pela QRI. Nesse sentido, propomos explorar e construir um sistema de governança multissetorial.

\section{Introducción}

El presente trabajo tiene como propósito ofrecer alternativas de gobernanza innovadoras para el emergente sector 4.0, o Cuarta Revolución Industrial en los países de América Latina, basadas en la experiencia reciente en Internet y en las industrias propias de otros países. Con ello se espera abreviar el tiempo y reducir el esfuerzo necesario para el 
apropiamiento social del conocimiento y su difusión en beneficio de la sociedad.

El desarrollo económico, social e industrial de los países latinoamericanos es heterogéneo y desigual en muchos sentidos. Coexisten en un mismo punto geográfico -y cada vez más descentralizado- desigualdad económica, graves carencias sociales, hambre, enfermedad, violencia y desesperanza con polos de desarrollo equiparables a los de países avanzados. Estos polos están interconectados con sus pares, clientes, y proveedores dentro y fuera del país a través de redes de colaboración y cadenas de suministro cada vez más complejas y basadas en una evolución incontenible de la tecnología que utilizan. No se trata sólo de fábricas sino de complejos económicos, educativos y sociales en los que concurren maquinaria e instrumentos, software y redes, y conocimiento y competencias avanzados que se despliegan también en el ámbito local. En numerosos países de la región se desarrollan clusters y otras figuras, no todas deliberadas, en los que ocurren rápidas transformaciones en la base industrial y la de conocimiento. Por ejemplo, sectores como el aeroespacial, el automotriz, el farmacéutico y el de materiales, están aprovechando tecnologías como la fabricación aditiva, la nanotecnología, la química avanzada, la biología molecular, la inteligencia artificial y avances en la logística. Estas también están afectando de manera creciente a la educación científica y tecnológica, la vinculación universidad-industria. Es innegable su influencia en el gobierno y la sociedad, y la vinculación entre los sectores privado, social, público y técnico o del conocimiento. El desafío para nuestros países consiste en asimilar rápidamente estas actividades en forma competitiva sin introducir nuevos y agravados desequilibrios en nuestras sociedades, no sólo de carácter económico sino también los ambientales y de género. Adicionalmente, es importante entender las particulares consecuencias no planeadas en el campo ético de las tecnologías con relación a, por ejemplo, la aplicación de herramientas de inteligencia artificial en diferentes ámbitos de la vida social, que es objeto de controversia a nivel mundial.

Los países en los que se ha originado esta revolución tecnológica de extenso impacto social han producido cambios en su gobernanza acompasados a su desarrollo. En los nuestros debemos encontrar formas propias para anticipar los cambios y mitigar sus consecuencias. Como se discutirá en el cuerpo del trabajo, las sociedades deben intervenir para que los cambios en educación, en la vinculación escuela-industria, en la regulación gubernamental, etcétera, no dependan solo de una decisión de los grupos dirigentes, ya que determinarán el futuro del trabajo y las condiciones para una vida digna.

El Foro Económico Mundial (o WEF por sus siglas en inglés) recomienda una gobernanza multisectorial; añadimos la participación de la sociedad civil y las comunidades expertas en forma explícita y con mayor ponderación, por las condiciones de las sociedades en desarrollo. Su participación cumple fines en dos direcciones: de "entrada" a los procesos alimentándolos con criterios diversos, como la no discriminación y la integración de toda la sociedad, y de "salida", como emisarios de las decisiones tomadas, para llevarlas a sus sectores y comprometerlos a actuar. Por ejemplo, los participantes de instituciones académicas no sólo mejorarán su enseñanza sino que introducirán laboratorios, planes de 
estudio, y modificaciones a los modelos de gestión de las propias instituciones, etc. Modelos como el Foro Consultivo Científico y Tecnológico (FCCyT) de México deben ser aprovechados y enriquecidos.

Los conceptos Cuarta Revolución Industrial (CRI), tecnología 4.0 o industria 4.0 son usados para describir una etapa de desarrollo tecnológico y social caracterizada por un salto cualitativo en la integración de cómputo, telecomunicaciones, automatización, operaciones, logística e integración de tecnologías, incluyendo las biológicas, hasta ahora dispersas, que da lugar a sistemas ciberfísicos que permitirán desarrollar nuevas capacidades y formas de insertar la tecnología tanto en la sociedad como dentro de organismos biológicos. El nombre Cuarta Revolución Industrial fue acuñado en el WEF con la intención de su presidente, Klaus Schwab, de atraer atención a las oportunidades que la conjunción de estas tecnologías genera para las sociedades tanto de los países industriales avanzados como de los países en otras etapas de desarrollo. El WEF destaca las posibilidades de reorganización de la producción que se abren también con las telecomunicaciones, los dispositivos móviles inteligentes, ampliamente difundidos entre la población, y la posibilidad de aplicar un enorme poder de procesamiento computacional a todas las tareas ${ }^{[1]}$.

Otros autores, como Tim Unwin (2019), consideran que estas categorías carecen de substancia y se trata ante todo de agrupamientos de moda con objetivos de publicidad y la promoción de una agenda ventajosa para un grupo de empresas y sus aliados en algunos gobiernos (véase Unwin 2009). En este trabajo mantenemos consciencia de esta visión crítica; utilizaremos la terminología de la CRI como una forma resumida de denominar a un proceso sustantivo, aunque no monolítico ni inexorable o incuestionable. Para nosotros se trata de tecnologías que recientemente tuvieron grandes avances por separado y que permiten producir resultados importantes cuando operan en conjunto. Si bien no existe un listado canónico sobre el conjunto específico de tecnologías que abarca la CRI, con frecuencia se incluyen las siguientes:

- Big data o análisis masivo de datos: aprovechamiento de grandes volúmenes de datos, generalmente en tiempo real o plazos muy cortos con base en la ciencia de datos, para analizar, dar sentido, y producir proyecciones y predicciones.

- Inteligencia artificial (IA): sistemas computacionales que permiten analizar grandes volúmenes de datos y a) extraer patrones difíciles de discernir por inspección humana, b) identificar excepciones a patrones cuya regularidad hace difícil discernir dichas excepciones por inspección humana y c) extrapolar a partir de los análisis a casos que no se utilizaron para calibrar los análisis. Misuraca y van Noordt (2020) mencionan tres componentes de la IA: percepción (la capacidad de una máquina o de un programa para entender impulsos provenientes de su entorno), razonamiento (capacidad de analizar y desprender inferencias de la información disponible) y acción (capacidad de que los sistemas de IA realizan tareas tanto en el plano digital como en el mundo físico). Entre los conceptos de IA en investigación, desarrollo y utilización más extendidos están el aprendizaje de máquina (ML 
por sus siglas en inglés), las redes neuronales (NN por sus siglas en inglés), el aprendizaje profundo y sistemas de lenguaje natural.

- Robótica: uso de máquinas que tienen grados altos de autonomía en la realización de sus tareas (robots en la manufactura, autotransportes autónomos, drones, etcétera). Estos robots pueden realizar trabajos similares a los que desempeñan los humanos o tener capacidades (fuerza) superiores.

- Fabricación aditiva: también conocida como tecnologías de impresión 3D, un dispositivo deposita sucesivamente capas de un material siguiendo un plano tridimensional seccionado en capas del espesor de las gotas del material.

Cada una de estas tecnologías, y su uso en conjunto, introducen cambios disruptivos y tienen consecuencias exponenciales, frente a las cuales las sociedades, especialmente aquellas que son fundamentalmente receptoras de estos desarrollos, deben plantarse una postura estratégica, que puede ir desde la pasividad, la asimilación o el fomento activo a la innovación. Este texto presenta, en su primer apartado, las implicaciones del acelerado avance tecnológico en términos de gobernanza de la innovación a la luz de experiencias del pasado. En la sección siguiente se exploran las implicaciones que la CRI tiene en términos de la relación entre gobierno y sociedad. La tercera parte se enfoca en ofrecer algunas alternativas para el diseño de un modelo de gobernanza de los mencionados desarrollos tecnológicos. Le siguen luego unas breves conclusiones.

\section{Gobernanza e innovación: los aprendizajes del pasado}

En la década de 1990 la innovación en Internet se tornó vertiginosa. Gobiernos y juristas debieron reconocer que la velocidad a la que se desarrollaban la tecnología y los cambios sociales resultantes se volvía inalcanzable y de un grado de granularidad demasiado fino para la capacidad normativa y legislativa de los mecanismos tradicionales de los parlamentos y del poder ejecutivo nacionales. Se caracterizó, además, por sus efectos transfronterizos que rebasan el alcance de los instrumentos nacionales y de cooperación internacional preexistentes. Se dio paso a conceptos, acuerdos, mecanismos e instituciones de gobernanza novedosos, en los que el Estado puede participar, pero no lo hace de manera exclusiva ni necesariamente con un poder decisorio final.

La gobernanza de Internet es ilustrativa para el presente estudio: dada su naturaleza en extremo descentralizada, su creación, puesta en marcha, operación y evolución requieren acuerdos entre partes muchas veces distantes entre sí en sentido geográfico o funcional tales como la sociedad civil organizada, ingenieros y abogados, empresas de distintos ramos, clientes, el gobierno, innovadores, y muchos más. Se requieren dichos acuerdos para la interconexión de redes, para contribuir a que éstas sean estables y robustas, para la seguridad de los sistemas conectados y de las propias redes, y para muchos otros fines. Bajo el mantra "la conectividad es su propio premio" muchos de estos acuerdos se toman 
entre pares, inicialmente de manera informal y, a lo largo de los años, mediante contratos multinacionales complejos. Sólo hay un recurso de naturaleza central, la coordinación en la asignación de los identificadores que deben tener valores únicos (nombres de dominio, direcciones numéricas o direcciones IP, y parámetros de los protocolos de la IETF). La coordinación de estos identificadores a nivel central (ya que se procede por delegaciones de autoridad) residió originalmente en el medio académico y de investigación de Estados Unidos, donde fueron creados los sistemas iniciales. La expansión de Internet más allá del ámbito gubernamental y académico generaría litigios costosos y responsabilidades a entidades académicas (algunas de ellas privadas) y al gobierno, que no podrían enfrentar. Por ello el gobierno de la UE decidió transferir a un organismo fuera del gobierno estas funciones de coordinación, a través de una convocatoria conocida como Green Paper. La reacción internacional a esta propuesta condujo a cambios de los que derivó una segunda convocatoria, esta vez con alcance global, llamada White Paper que resultó en la creación de ICANN (Internet Corporation for Assigned Names and Numbers).

La reacción de la muy lucrativa industria de registro de nombres de dominio fue inicialmente oponerse y cabildear intensamente. Cuando, más tarde, fue evidente que ICANN no desaparecería, esta industria trató de debilitar a la organización mediante litigios costosos y campañas dirigidas a reducir su legitimidad. Asuntos tales como los mecanismos para evitar la piratería de nombres de marcas han sido especialmente álgidos (Apke, 2003). Como resultado, se establecieron mecanismos de solución de disputas (Uniform DomainName Dispute-Resolution Policy) a partir de acuerdos entre diversos actores, que han dado lugar a que la organización sea estable, robusta y cada vez más independiente. Procesos similares ocurren en otros sectores como es el caso de la regulación de impacto ambiental, donde se ha tratado de evitar la captura y deslegitimación de los mecanismos, estableciendo algún tipo de equilibrio dinámico multisectorial entre los actores, con diferencias a niveles nacionales y con acuerdos globales (Oreskes y Conway, 2010). Ejemplo de esto fue la exitosa reducción del agujero de ozono mediante la disminución de las emisiones que lo causaban.

Algunos temas fundamentales de la CRI están en etapas similares a las iniciales e intermedias de los ejemplos ofrecidos. La robótica está fuertemente regulada desde el punto de vista de seguridad e higiene industrial. El procesamiento de datos personales cuenta ya con un marco regulatorio en muchos países, aunque no es el caso para sus usos avanzados en el perfilamiento en redes sociales y el llamado capitalismo de vigilancia (uso comercial de la información de las y los consumidores). La inteligencia artificial es objeto de atención por sus posibles efectos negativos e indeseables mediante la formulación de marcos éticos que buscan la reducción de sesgos discriminatorios, promueven la transparencia y la explicabilidad de los algoritmos utilizados, y limitan o frenan por completo los usos más peligrosos, como las armas letales totalmente autónomas. En una etapa mucho más abierta y exploratoria encontramos los debates sobre el futuro del trabajo consecuencia de la automatización de las tareas de mayor demanda intelectual. Los marcos éticos que están en desarrollo son muy diversos; algunos son deontológicos, otros se basan en la virtud y otros 
más son utilitaristas, amén de algunas combinaciones y mezclas. El Institute of Electrical and Electronics Engineers (IEEE) ha hecho un inventario de bases éticas que incluyen las del budismo y las del confucionismo, con referencia específica al desarrollo de la inteligencia artificial pero que puede extenderse a otros campos de la CRI (Shahriari y Shahriari, 2017).

En suma, de la construcción de la gobernanza de Internet hemos aprendido que:

1. Es necesario incluir a todos los posibles actores, sea que los mueva el interés económico o político, la defensa de principios que les son caros, o alguna combinación de estos factores.

2. Los mecanismos, acuerdos, e instituciones deben orientarse a resolver problemas bien definidos (enfoque que llamamos heurístico).

3. Los mecanismos, acuerdos e instituciones deben contar con estructuras y capacidades proporcionales a la magnitud del problema que resuelven y tener legitimidad para que las partes interesadas participen en la construcción de sus políticas y normas, financien su operación y acaten sus resoluciones.

4. Los foros, acuerdos e instituciones deben contar con mecanismos de transparencia, rendición de cuentas, apelación de decisiones y reversión de estas en su caso.

Estas reglas se han extraído de la gobernanza de ámbitos tan diversos como el medio ambiente, las finanzas, el deporte y, desde luego, las tecnologías de información e Internet. Como ha pasado con muchas otras innovaciones, observamos tanto las tendencias preexistentes que se preservan como los cambios, a veces disruptivos, que conlleva la nueva tecnología.

\section{Relaciones gobierno-tecnología-sociedad en la Cuarta Revolución Industrial}

Como se ha descrito en la sección anterior, la CRI implica un reto regulatorio enormemente complejo tanto por la naturaleza misma del fenómeno como por la necesidad de desarrollar capacidades novedosas tanto en el sector público, como en las empresas y en la sociedad en general. En esta sección, sin embargo, nos centramos en otros tres efectos de la CRI que es necesario considerar: en primer lugar, los impactos que sobre las relaciones gobiernosociedad pueden tener estas tecnologías - particularmente frente al potencial avance de nuevos mecanismos de dirección social (steering) como son la gobernanza algorítmica一; en segundo lugar, el gobierno puede ser usuario de las tecnologías 4.0 en la prestación de servicios públicos y sus potenciales consecuencias y, tercero, los efectos de la CRI en las formas de organización y funcionamiento de las administraciones públicas. Se analizan cada uno a continuación. 


\section{Efectos en la relación Estado-sociedad}

La incorporación de tecnologías en la gestión de los asuntos públicos no es nueva. Sin embargo, en el caso de la CRI, esta se vuelve un tema estratégico, que impacta la forma en que se relaciona el Estado y la sociedad. En el caso de los países de América Latina, las desiguales capacidades estatales, donde los esquemas de gestión patrimonialistas conviven con sistemas tecnológicos modernos, donde el estado de derecho coexiste con patrones informales y tradicionales de ejercicio del poder, la CRI tiene dos caras: la de reforzar positivamente la imparcialidad, la eficiencia y la oportunidad en la prestación de servicios, y, por otro lado, la de reproducir y acentuar brechas y desigualdades sociales, y generar así problemas éticos y hasta conflictos políticos al redefinirse los equilibrios de poder entre los diferentes sectores sociales. Simplemente, el desplazamiento masivo de trabajadores de baja calificación en los sectores privado y público, así como el cierre de horizontes de oportunidad para ciertos grupos sociales como las y los jóvenes o las mujeres, puede traer nuevas tensiones a la región ${ }^{[2]}$.

Existe un consenso creciente que considera que tecnologías como la inteligencia artificial, la robótica y la Internet de las cosas replantearán el papel del Estado y las formas en que las personas se relacionarán con el sector público (Ramió, 2019 y Hanna, 2017). Por ejemplo, Criado introduce el concepto de gobernanza pública inteligente, que entiende como un nuevo enfoque de reforma de la gestión pública. Retoma la idea central de que en el mundo contemporáneo los gobiernos carecen de las capacidades y los recursos suficientes para atender problemas públicos complejos, por lo que se hace necesario establecer alianzas con los sectores privado y social para compartir recursos y responsabilidades. Adicionalmente, la gobernanza inteligente implica apoyarse en las nuevas tecnologías para facilitar formas de interacción novedosas, donde el gobierno fomenta la cocreación de valor público y la innovación (Criado, 2016). En sentido similar, Dunleavy et al. (2006) hablan de una gobernanza de la era digital, donde la documentación en papel es sustituida por la electrónica, los procesos de gobierno están plenamente integrados y flexibilizados para hacer frente a necesidades cambiantes de sus usuarios, se aprovecha la colaboración intersectorial de manera intensiva y la información se disemina por múltiples canales en formatos abiertos.

Otros autores advierten sobre las expectativas poco realistas respecto a los resultados de estas herramientas tecnológicas. Schou y Hjelholt (2018) señalan que es necesario entender la digitalización del sector público en el contexto más amplio de un sistema económico que ha transitado un estado de bienestar keynesiano al estado de la competitividad contemporáneo, y que hace énfasis en la necesidad o inevitabilidad de la competencia entre diferentes naciones que fundan su desarrollo en el mercado liberalizado. Así, más que un camino lineal, la forma específica en que las administraciones públicas adoptarán las tecnologías 4.0 dependerá, por una parte, de las presiones externas que se generan sobre las administraciones nacionales, y por otra, por condicionamientos o herencias específicas que faciliten o compliquen estos procesos de asimilación, tales como la adecuación de la 
legislación vigente, las estructuras organizativas preexistentes, las capacidades gerenciales disponibles, y el perfil y la motivación de los servidores públicos (Lee y Reed, 2015). En este sentido, América Latina y el Caribe presenta retos particulares dado el variopinto panorama de las capacidades estatales para liderar las transformaciones derivadas de la CRI. Sólo por citar un ejemplo, Oxford Insights y el International Research Development Centre (IDRC) publican cada dos años el Índice de Preparación de los Gobierno para la Inteligencia Artificial, que otorga puntajes de 0 a 100 a partir de valorar dimensiones como disponibilidad de datos, infraestructura, capital humano, capacidad de innovación, gobernanza y ética, entre otras. Nuestra región está entre las más atrasadas, con enorme variabilidad en las calificaciones: en la edición 2020 del índice, mientras Uruguay liderea con una calificación de 55.57, Haití obtuvo apenas 21.11 puntos. En términos de políticas, mientras Colombia y Uruguay cuentan con sólidas estrategias de desarrollo de innovación digital, otros países como Argentina, Brasil, Chile y México aún no cuentan con ninguna. (Shearer, Stirling y Pasquarelli, 2020).

El potencial positivo de las tecnologías es aprovecharlas para, a partir de la vinculación entre espacios virtuales, máquinas y sensores, se puedan construir gobiernos smart (Criado, 2016). También, se habla de que estos desarrollos llevan la transparencia a un nuevo nivel, en la medida en que el compartir información dentro y fuera del gobierno se convierte en el centro de un sistema social de inteligencia que, con base en las tecnologías, permita una participación ciudadana más amplia y eficaz en la gobernanza (Hanna, 2017 y Noveck, 2009 y 2015). En particular, el uso de redes sociales puede permitir el flujo de un conocimiento difícil de codificar y que reside en redes de expertos del sector público y de fuera de él (Mergel, 2010). Por otra parte, la creación de las llamadas redes colaborativas de datos (data collaboratives) tienen el objetivo de hacer accesibles bases de datos que están dispersas o restringidas (derechos de propiedad), y así puedan ser utilizadas por los diseñadores de soluciones (por ejemplo, cruce de fuentes gubernamentales y no gubernamentales de información) para atender asuntos de interés general (Susha et al., 2017).

Adicionalmente, para evitar abusos o la reproducción de desigualdades (como las derivadas de las brechas digitales), es importante cerrar el déficit democrático que puede introducirse en las decisiones sobre el uso de estas tecnologías por parte del sector público. En este sentido, el uso de programas de reconocimiento facial o el rastreo georreferenciado desde aparatos como los teléfonos celulares tienen aplicaciones positivas, de valor público, pero también pueden ser utilizadas de manera abusiva tanto por actores públicos como privados. Como ejemplos citamos los sistemas de puntuación para el control social, el software de espionaje (Marczak, 2018), y el uso de bots desde el gobierno para alterar las tendencias en redes sociales, con el objetivo de generar aparentes consensos con relación a ciertos temas o personajes, vulnerando el derecho a la libre circulación de las ideas (Treré, 2016). En países receptores estos riesgos se agravan, en la medida en que los desarrollos tecnológicos pueden estar diseñados según referentes y necesidades de los países centrales, resultando inadecuados o contraproducentes en los contextos locales. Aunado con lo anterior está el 
asunto de las jurisdicciones legales, pues frecuentemente los términos de uso de las nuevas tecnologías incluyen el acudir forzosamente a instancias en el extranjero para dirimir querellas o establecer responsabilidades.

\section{Gobierno como usuario y cambios al interior de la AP}

Otro aspecto concreto en que la CRI tendrá efectos en el gobierno es en la prestación de servicios públicos. La bibliografía refiere algunos beneficios potenciales como concentrar a las personas servidoras públicas en trabajos de alto valor agregado, reduciendo la necesidad de realizar tareas manuales y repetitivas, capacidad de generar soluciones y servicios cortados a medida de las necesidades de los usuarios, capacidad de tomar decisiones mejores gracias a la disponibilidad de acceso a evidencia en tiempo real, entre otros. En particular, las tecnologías de IA y smart son ejemplos de este potencial. Entre los beneficios esperados de estas tecnologías están el mejorar la calidad del procesamiento de información, haciéndola más rápida y precisa y, el tratamiento acelerado de los casos o solicitudes, la mejora de las cargas de trabajo (Wirtz y Müller, 2018, y Martínez Martínez, 2019). Un caso en este sentido es la creación de sistemas conversacionales automatizados (chatbots) que permiten interactuar con las personas que utilizan dispositivos electrónicos (ADIP, 2019 y Desouza et al., 2020). Otras oportunidades se refieren al aprovechamiento de cantidades masivas de datos para mejorar la prestación de servicios como el transporte público, la provisión de agua o los apoyos a los agricultores para fomentar su productividad (Ramió, 2019). Finalmente, también avanza la automatización de tareas complejas, como es la redacción de proyectos de sentencia en el ámbito de la impartición de justicia (Estévez et al., 2020).

En este sentido, nuevamente hay importantes riesgos de reproducción o creación de nuevos ejes de discriminación, como han mostrado tanto el programa de reconocimiento facial usado por la policía de Chicago, que señalaba a personas inocentes (Desouza et al., 2020), y el caso del algoritmo COMPAS desarrollado por una empresa privada y utilizado en los Estados Unidos para dictar decisiones judiciales, que discriminaba según variables socioeconómicas y raciales. En este caso, el hecho de que el propio algoritmo está protegido hace imposible saber qué tipo de información y ponderaciones hace para arribar a sus conclusiones (Ramió, 2019). Es importante diferenciar los efectos discriminatorios originados por los sistemas de aquellos que surgen porque los datos de entrada codifican conductas discriminatorias preexistentes.

Finalmente, en términos organizativos, los países más proactivos en este campo han incorporado a sus estructuras nuevos puestos como los Chief Information Officers (CIO) o, más recientemente, los Chief Data Officers (Aron y Waller, 2014). En España y en algunos países más, la transformación digital se atiende a nivel de ministerios especializados. En el caso más desarrollado en nuestra región, Uruguay creó la Agencia de Gobierno Electrónico y Sociedad de la Información (AGESIC) que está encargada de llevar adelante la estrategia digital del país y asume también el papel de CIO (Velasco, 2019). Este ha sido un caso 
bastante exitoso en la región, que ha permitido al país colocarse entre aquellos que mejor han conducido su inserción en el contexto de la CRI. Es también un caso que llama reflexionar sobre cómo gobernar estos procesos de manera activa y por medio de la integración de diversos actores, en clave de gobierno abierto.

Otro reto de los procesos de automatización y la introducción de robots es la potencial reducción drástica de plantillas laborales: de acuerdo a la consultora Deloitte, en el Reino Unido desaparecerán 861.000 empleos para el año 2030. Ramió (2019), refiriéndose al sector público español, señala áreas más susceptibles a sufrir el desplazamiento de la fuerza laboral en el sector público ya no sólo a los niveles operativos, sino a empleados de tareas de vigilancia, sanidad, trabajo social, entre otros, lo que estima que representará alrededor de 10\% del empleo público. Gorriti Bontigui (2018) hace un análisis de la demanda futura de los puestos de trabajo, identificando como los de mayor crecimiento a los relacionados a las ciencias, las tecnologías, la ingeniería y las matemáticas, y que son de carácter profesional al desempeñar tareas complejas. Aunado lo anterior a otros cambios concurrentes puede producirse un conjunto de personas trabajando por proyectos con esquemas de prestaciones laborales mínimos o inexistentes, engrosando una especie de ejército de burócratas de reserva (Jiménez Ascencio, 2017). De manera paradójica, se trata de una burocracia sin burócratas, donde los usuarios se vuelven sus propios administradores: self-service bureaucracy (Ramió, 2019 y Schou y Hjelholt, 2018). Para los países de América Latina el reto es mayor si se considera la diversidad de mecanismos de reclutamiento de personal público, que va desde servicios civiles consolidados hasta el mantenimiento de prácticas patrimonialistas como los sistemas de botín, por un lado, y la existencia de amplias capas del funcionariado que cuentan con protecciones laborales y que están respaldados por sindicatos fuertes, por el otro. Conducir una estrategia de desarrollo de personas que sea coherente frente a los cambios introducidos por la CRI se vuelve aún más complejo.

Finalmente, Ramió (2019) señala que uno de los riesgos de una actitud pasiva del sector público frente a la CRI es terminar en situación de dependencia excesiva de desarrollo pensados desde y para el sector privado $-\mathrm{y}$, agregamos, desde los países centrales-, lo que puede reducir su utilidad para apoyar las actividades del sector o público o, en el peor de los casos, terminar por generar resultados que erosionan la confianza ciudadana en el gobierno y sus organizaciones. De esta manera, la generación de desarrollos propios no es un asunto meramente simbólico, sino que es un planeamiento necesario para asegurar la eficacia de las herramientas en los contextos y marcos legales latinoamericanos, como la incorporación de valores y principios adecuados a nuestros contextos sociales. La generación de desarrollos propios, además, es un acto educativo primordial para formar personas que entiendan en profundidad las innovaciones.

\section{Alternativa: gobernanza multisectorial}

El espectro de opciones descrito hasta aquí nos lleva a proponer que en la CRI existen 
algunas necesidades claras y apremiantes de gobernanza y que los gobiernos y las herramientas propias del Estado son insuficientes para producirla por sí solos, por no contar con la velocidad, la capacidad de respuesta, la granularidad en tiempo y en materia de trabajo, el expertise técnico, legal, organizacional y ético, y el carácter rizomático de la participación necesarias.

La gobernanza multisectorial (MSH por multistakeholder) ha sido una respuesta que se ha dado a situaciones de similar complejidad. Es nuestra opinión que representa una alternativa que debe adoptarse plenamente y contar con el respaldo del Estado para hacer que sea viable y efectiva, con los ajustes necesarios para adaptarla a las necesidades de cada caso particular.

Se ha discutido por largo tiempo el papel del Estado ante la innovación tecnológica. En un trabajo particularmente interesante, Lyall y Tait (2019), proponen el concepto de "gobernanza tentativa", para el caso particular de las ciencias de la vida, como gobernanza en un período de transición entre la aparición de las innovaciones y su incorporación plena a la sociedad. De manera complementaria, Borrás y Edler (2020) revisan los posibles papeles del Estado y los listan como: observador, vigía o alertador (warner), mitigador, oportunista, facilitador, usuario líder, promotor, moderador, iniciador, garante y vigilante (watchdog). Todos estos roles están disponibles para el Estado en los países en los que se lleva a cabo el desarrollo original de la ciencia y la tecnología. En países dependientes o en desarrollo, las capacidades de iniciador y otras están inhibidas, y los interesados en el statu quo presionan para que algunos de estos roles se viertan hacia impedir el desarrollo e implantación de innovaciones que pueden dar ventajas competitivas a nuevos actores. Los temores de sectores de la sociedad pueden sumarse y, envueltos en invocaciones al principio precautorio, pueden exacerbar los roles de garante, vigilante y moderador. Por otra parte, el rol de usuario líder se puede ver minado por falta de capacidades y por distorsiones en los procesos de compras del sector público.

Desde el WEF (Elmi et. al, 2018) se propone una gobernanza “ágil”, distinta del paradigma "ágil" de programación de computadoras, basada en opciones como autorregulación de la industria, creación de superreguladores, formulación de normas éticas, creación de ecosistemas de gobernanza colaborativa y construcción de confianza en la ciencia y la tecnología. Retomamos en particular la creación de ecosistemas de gobernanza colaborativa, considerando además que la autorregulación de la industria tiene limitaciones severas, especialmente en países que no son los originadores de las empresas y en los que la competencia en el mercado no tiene el mismo poder regulatorio que en los países centrales. Inspiradora resulta la imagen de involucramiento "río arriba" (up-stream engagement) propuesto por Rodemeyer, Sarewitz y Wilsdon (2005), que se refiere a un proceso de diálogo constante con actores sociales, iterativo, en los procesos de innovación.

Nos preocupa además que sin una gobernanza adecuada los países latinoamericanos puedan quedarse en escenarios de atraso y de seguidores, antes que adoptar una visión 
integral de futuro, que incluya los desarrollos de la CRI a un proyecto de desarrollo nacional (Cabrero et al., 2020). Es indispensable que los gobiernos de la región actúen en función de un sólido compromiso a largo plazo con la innovación dejando grandes espacios a la colaboración horizontal y las iniciativas espontáneas de los sectores. La gobernanza multisectorial puede ayudar a definir las brechas en que el gobierno u otros actores deban incidir más agudamente.

Podemos explorar la propuesta más a fondo sobre un tema específico, el de la Internet de las cosas (IoT por sus siglas en inglés). En la IoT, tanto para el consumidor como la industrial, intervienen fabricantes de dispositivos de uso general, dispositivos y sistemas de uso industrial, comercial o gubernamental, y dispositivos para el consumidor; telecomunicaciones de largo, mediano y corto alcance, con exigencias diferentes de anchura de banda, latencia y disponibilidad, lo que a su vez implica diferencias en el acceso libre o regulado al espectro radioeléctrico, en distintas bandas atendiendo a sus propiedades; software en la "orilla", en la "nube" y en la "niebla". En una posible implementación, las empresas operadoras de redes pueden vender como servicio "rebanadas" de red (slices), con todos los componentes dentro de un subsistema lógico y con posibles violaciones a los principios de separación de capas de Internet y de neutralidad de la red en Internet. Sistemas y servicios administrados, cadena de suministro hacia el dispositivo y desde su implantación, y empresas que ofrecen servicios integrados con todo lo anterior. Para ello además se disputan estándares técnicos en una tensión entre interoperabilidad y control/exclusividad, alianzas de grandes empresas transnacionales, intervenciones de grandes potencias con impacto geopolítico global, y, por último, pero no de menor importancia, ciudadanos, que ponen en manos de estos sistemas su seguridad cibernética y también física.

La alternativa que proponemos es la formación de una arquitectura de organizaciones y mecanismos que combinen la producción de acuerdos entre los fabricantes y operadores; la participación ciudadana; la educación de consumidores y todos los otros miembros de la cadena de suministro; y la autoridad de los órganos del Estado, desde el fomento del desarrollo económico hasta las fuerzas del orden, con una fuerte interconexión internacional. Además, las administraciones públicas de los países que no son las sedes principales de estos desarrollos tecnológicos deben:

1. Intensificar la educación en matemáticas, ciencia, tecnología, computación y capacidad de innovación, sin descuidar la lógica, la ética, las humanidades, y las artes.

2. Fomentar el desarrollo tecnológico con una combinación de flexibilidad y planificación a futuro, en forma descentralizada al interior del país.

3. Hacer que miembros del Estado - los poderes ejecutivo, legislativo y judicial, y los órganos reguladores autónomos- adquieran conocimientos y experiencias en los mecanismos multisectoriales, mediante educación y fomentando su participación directa en los foros internacionales no multilaterales "clásicos". 
4. Convocar a amplias reuniones multisectoriales al interior del país y por regiones o grupos de afinidad, explotando las tecnologías de comunicación, en un ambiente abierto a las ideas y la innovación, que el Estado debe después conducir a materializar en programas concretos.

5. Acompañar a la sociedad en el proceso de construcción de mecanismos multisectoriales propios y favorecer su integración con los correspondientes en los planos internacionales global y regional.

6. Identificar su posible participación en cada proceso y fase de acuerdo con los roles descritos y convocar expertise y participación alrededor de ellos.

Haremos referencia ahora solamente a IoT por brevedad, pero la formulación es extensible a otras de las tecnologías y se integrará de acuerdo con los problemas y conjuntos de stakeholders pertinentes, los que pueden presentar una amplia variación geográfica. En el ejemplo de IoT algunos pasos concretos de la construcción de gobernanza incluirían:

\section{Asignación de espectro radioeléctrico.}

2. El uso de IoT de consumidor conlleva riesgos de seguridad para los propios usuarios de dispositivos y para los de otras partes de las redes que pueden ser atacadas mediante la penetración y usurpación del control de estos dispositivos. Está entonces en interés de la sociedad poder elevar constantemente sus niveles de seguridad mediante confinamiento en redes detrás de cortafuegos, actualizaciones de firmware y software, forzamiento de contraseñas seguras y cambios frecuentes, y otras medidas. En los países de la región no cuenta con órganos de normalización con autoridad suficiente para mandatar eficazmente estas características; más aún, la homologación de estándares y normas con los países del Tratado de Libre comercio entre México, Estados Unidos y Canadá (T-MEC) reduce de facto las capacidades del Estado en la materia. La presión de algunos grupos de consumidores tendría que articularse a nivel del área de América del Norte para inducir cambios en las normas técnicas y litigios estratégicos que asignen responsabilidades a empresas y operadores que no fuercen las reglas descritas. La actual situación de capitulación normativa, en la que las normas técnicas son elaboradas esencialmente por la industria, debe cambiar a una de mayor intervención de la comunidad experta y la sociedad.

3. En IoT industrial aparece un conjunto diferente y complementario de riesgos, como la posible pérdida de puestos de trabajo de calidad debido a la automatización avanzada, la pérdida de control y autonomía en el trabajo debido a que este cade vez más esté dirigido por los resultados de análisis de datos masivos, etcétera. En este tema son los sindicatos y las organizaciones de la sociedad civil las principales entidades capaces y con interés -incluido el interés jurídico acreditable- en forzar una mayor intervención social en la gobernanza de este sector. Sistemas de impacto más extenso, como los vehículos autónomos, requieren una construcción de normas ampliamente participativa.

4. Mecanismos de gobernanza multisectorial como los esbozados en estos párrafos corren 
un alto riesgo de dejarse paralizar por miedo a lo desconocido, es decir, por una aplicación excesiva del principio precautorio, desde luego mucho más intensa que en los países de origen de las tecnologías. Por ello una acción urgente de gobernanza será la educación, desde el impulso al pensamiento computacional y a las áreas STEM en la educación elemental, hasta campañas que produzcan un aumento sustancial en el aprecio de la innovación.

La arquitectura institucional que visualizamos reunirá organizaciones existentes, como los Consejos Técnicos y de Vinculación de algunas escuelas, con otras por crear, como foros regionales. Otra evolución posible es enriquecer estructuras como los Consejos Directivos de Clusters, como los Aeroespaciales en el Noroeste de México.

\section{Conclusiones}

A partir de una descripción de los conceptos generales contenidos en la propuesta de Cuarta Revolución Industrial del Foro Económico Mundial y posibles formas de implantación de esta, hemos señalado retos para los países de América Latina en su aplicación en la administración pública y en su gobernanza. El gobierno puede establecer muchos tipos de relaciones con estas tecnologías 4.0: puede ser un promotor activo de la innovación o ser más un espectador, con múltiples otras opciones intermedias. Igualmente, puede ser un promotor del aprovechamiento de los avances tecnológicos para replantearse en alguna medida la relación con la sociedad, abriéndose retos de transparencia y rendición de cuentas; y puede ser proactivo o reticente a usar estas tecnologías para mejorar la prestación de servicio y reorganizarse internamente, con los riesgos políticos y éticos de una burocracia automatizada. Todas estas facetas implican cambio de tal velocidad y granularidad, que los esquemas tradicionales de mando y control no parecen adecuados para enfrentar los retos que presenta la CRI.

Proponemos explorar y construir un sistema de gobernanza multisectorial, federado, con superposiciones entre los mandatos de los distintos mecanismos que puedan componerlo, transnacional, y cuyos componentes integrarán las necesidades de regulación sobre equipos físicos, software, sistemas de toma de decisiones, mecanismos de asignación de espectro radioeléctrico, reglas de operación de empresas y mercados de redes de telecomunicaciones, mecanismos para el tratamiento de asuntos laborales, y otros que pudieran ser requeridos. Este complejo sistema no debe ser diseñado de arriba hacia abajo sino emerger a partir de necesidades concretas y co participación efectiva de interesados y competentes, favoreciendo al máximo y en equilibrio, los derechos humanos y la innovación abierta.

\section{Bibliografía}

- Agencia Digital de Innovación Pública. (24 de marzo de 2019). La ADIP y el Gobierno de la 
Ciudad presentan Victoria, el robot que colaborará en la atención de la ciudadanía. https://adip.cdmx.gob.mx/comunicacion/nota/la-adip-y-el-gobierno-de-la-ciudad-presentan-vi ctoria-el-robot-que-colaborara-en-la-atencion-de-la-ciudadania

- Apke, T. M. (2003). Legal strategies in resolving domain name disputes. Industrial Management \& Data Systems, 103(5), 332-338.

- Aron, D., \& Waller, G. (2014). Taming the digital dragon: The 2014 CIO agenda. Gartner.com, 12.

- Borrás, S., \& Edler, J. (2020). The roles of the state in the governance of socio-technical systems' transformation. Research Policy, 49(5), 103971.

- Cabrero Mendoza, E., Carreón, V. \& Guajardo Mendoza, M. (2020) México frente a la sociedad del conocimiento. Siglo XXIEditores / CIDE

- Criado, J. I. (2016). Gobernanza inteligente, innovación abierta y tecnologías sociales en unas administraciones públicas colaborativas. ¿Hacia un cambio de paradigma en la gestión pública?. Nuevas tendencias en la gestión pública: Innovación abierta, gobernanza inteligente y tecnologías sociales en unas administraciones públicas colaborativas (pp. 22-48).

- Davis, N. (enero de 2016). What is the fourth industrial revolution? World Economic Forum.

- Desouza, K. C., Dawson, G. S., \& Chenok, D. (2020). Designing, developing, and deploying artificial intelligence systems: Lessons from and for the public sector. Business Horizons, 63(2), 205-213.

- Dunleavy, P., Margetts, H., Bastow, S., \& Tinkler, J. (2006). New public management is dead-long live digital-era governance. Journal of public administration research and theory, 16(3), 467-494.

- Elmi, N., Broekaert, K., \& Larsen, A. M. E. (2018, January). Agile Governance: Reimagining Policy-Making in the Fourth Industrial Revolution. White Paper. World Economic Forum, January.

- Estevez, E., Fillotrani, P., \& Lejarraga, S. L. (2020). PROMETEA: Transformando la administración de justicia con herramientas de inteligencia artificial. BID Invest. Recuperado de http://dx. doi. org/10.18235/0002378.

- Gorriti Bontigui, M. (2018). Los puestos de trabajo del futuro en el sector público. https://rafaeljimenezasensio.files.wordpress.com/2019/04/los-puestos-de-trabajo-del-futuro-e n-el-sector-pucc81blico_gorriti_marzo_2019.pdf

- Hanna, N. K. (2017). How Can Digital Technologies Improve Public Services and Governance?. Business Expert Press. 
- Harari, Y. N. (2016). Homo Deus: A brief history of tomorrow. Random House.

- Jiménez Asensio, R. (2017). El empleo público ante la digitalización y la robótica. online]. Blog La Mirada Institucional, 2.

- Lee, J., \& Reed, B. J. (2015). From paper to cloud. Public Administration Evolving (pp. 159-192). Routledge.

- Lyall, C., \& Tait, J. (2019). Beyond the limits to governance: new rules of engagement for the tentative governance of the life sciences. Research Policy, 48(5), 1128-1137.

- Marczak, B., Scott-Railton, J., McKune, S., Abdul Razzak, B., \& Deibert, R. (2018). HIDE AND SEEK: Tracking NSO Group's Pegasus Spyware to operations in 45 countries.

Martínez Martínez, R. (2019). Intel-ligència artificial des del disseny. Reptes i estratègies per al compliment normatiu (ES-EN). Revista catalana de dret públic, (58), 64-81.

- Mergel, I. (2010). Government 2.0 Revisited: Social Media Strategies in the Public Sector. PA Times, American Society for Public Administration, 33(3), 7-10.

- Misuraca, G., \& Van Noordt, C. (2020). AI Watch-Artificial Intelligence in public services: Overview of the use and impact of AI in public services in the EU. JRC Working Papers, (JRC120399).

- Noveck, B. S. (2015). Smart citizens, smarter state. Harvard University Press.

- Noveck, B. S. (2009). Wiki government: How technology can make government better, democracy stronger, and citizens more powerful. Brookings Institution Press.

- Oreskes, N., \& Conway, E. M. (2010). Merchants of doubt: How a handful of scientists obscured the truth on issues from tobacco smoke to global warming. Bloomsbury Publishing.

- Pisanty, A. (2016). The vexing problem of oversight and stewardship in Internet governance, . The Working Group on Internet Governance - 10th Anniversary Reflections, 189-207.https://www.apc.org/en/system/files/IG_10_Final.pdf

- Pisanty, A. (21 de mayo de 2020). Open Internet governance: The 6F framework and COVID-19. Medianama.

https://www.medianama.com/2020/05/223-open-internet-governance-6f-framework/

- Poole, S. (6 de enero de 2017). The Fourth Industrial Revolution review - adapt to new technology or perish. The Guardian.

https://www.theguardian.com/books/2017/jan/06/the-fourth-industrial-revolution-by-klaus-sc hwab-review

- Ramió, C. (2019). Inteligencia artificial y administración pública: robots y humanos compartiendo el servicio público. Los Libros de la Catarata. 
- Schou, J., \& Hjelholt, M. (2018). Digitalization and public sector transformations. Springer.

- Schwab, K. (2017). The fourth industrial revolution. Currency.

- Shahriari, K., \& Shahriari, M. (2017). IEEE standard review-Ethically aligned design: A vision for prioritizing human wellbeing with artificial intelligence and autonomous systems. In 2017 IEEE Canada International Humanitarian Technology Conference (IHTC) (pp. 197-201). IEEE.

- Shearer, E., Stirling, R. \& Pasquarelli, W. (2020). Government AI Readiness Index 2020.

- Susha, I., Janssen, M., \& Verhulst, S. (2017). Data collaboratives as a new frontier of crosssector partnerships in the age of open data: Taxonomy development.

- Treré, E. (2016). The dark side of digital politics: Understanding the algorithmic manufacturing of consent and the hindering of online dissidence. IDS Bulletin, 47(1).

- Unwin, T. (23 de marzo de 2019). 5 Problems with 4th Industrial Revolution - Your Weekend Long Reads. ICT Works.

https://www.ictworks.org/problems-fourth-industrial-revolution/\#.YFAKy68zaUk

- Velasco Sánchez, E. (2019). Dirigir la Revolución Digital en el sector público. Nuevas estructuras organizativas y perfiles directivos. Buen Gobierno, (27), 1-24.

- Wirtz, B. W., \& Müller, W. M. (2019). An integrated artificial intelligence framework for public management. Public Management Review, 21(7), 1076-1100.

\section{Acerca de los Autores}

Alejandro Pisanty Baruch: es profesor de carrera de la Facultad de Química de la UNAM. En la UNAM ha sido también Secretario del Consejo Asesor de Cómputo, Coordinador de Universidad Abierta y Educación a Distancia, y Director General de Servicios de Cómputo Académico. Preside la Sociedad Internet de México. En el ámbito internacional ha sido miembro de los Consejos Directivos de ICANN (Internet Corporation for Assigned Names and Numbers, del cual fue además vicepresidente por seis años) y de la Internet Society; miembro del Grupo de Trabajo para la Gobernanza de Internet, en la ONU, y en la misma ONU miembro del Grupo Asesor del Foro sobre Gobernanza de Internet. Además de su labor académica e internacional, ha realizado labores de consultoría en Internet, educación a distancia y tecnologías de la información, y es un activo participante de la comunicación sobre estos temas en medios sociales, en los cuales co-lideró la campaña "Internet Necesario" en 2009. En otros temas, destaca su participación en el proyecto "Juicio en Línea" del Tribunal Federal de Justicia Fiscal y Administrativa y otras colaboraciones con la Asociación Mexicana de Impartidores de Justicia. Fue miembro del Consejo Consultivo de la 
Comisión Federal de Telecomunicaciones.

Dr. Ernesto Velasco Sánchez: es Director General de Consultores en Gestión Pública y Social, S.C. Doctor en Gobierno y Administración Pública por la Universidad Complutense de Madrid, cuya tesis sobre rendición de cuentas con base en resultados y relaciones intergubernamentales recibió la distinción Cum Laude. Cuenta con una maestría en Gestión Pública por la Universidad de Birmingham y es licenciado en Administración Pública por El Colegio de México. Se especializa en temas de gestión de organizaciones públicas y sin fines de lucro, gobierno digital, evaluación de políticas y procesos deliberativos. Fue miembro del Panel Internacional de Expertos del Mecanismo Independiente de Revisión de la Alianza para el Gobierno Abierto (AGA) y facilitó procesos de gobierno abierto en Costa Rica, Ecuador, México, Panamá y Trinidad y Tobago. Cuenta con más de veinte publicaciones en temas de gestión pública, gobierno abierto, gobierno digital y evaluación. Es presidente del Consejo Directivo de Fundar, Centro de Análisis e Investigación, uno de los 10 mejores think tanks de América Latina.

\section{Notas}

La definición original de Schwab y el WEF es la siguiente: "The Fourth Industrial Revolution can be described as the advent of 'cyber-physical systems' involving entirely new capabilities for people and machines. While these capabilities are reliant on the technologies and infrastructure of the Third Industrial Revolution, the Fourth Industrial Revolution represents entirely new ways in which technology becomes embedded within societies and even our human bodies. Examples include genome editing, new forms of machine intelligence, breakthrough materials and approaches to governance that rely on cryptographic methods such as the blockchain." En https://www.weforum.org/agenda/2016/01/what-is-the-fourth-industrial-revolution/

En este sentido, nuestra región puede ser una de las más afectadas por el fenómeno de las clases inútiles (useless classes) a la que se ha referido Yuval Noah Harari (2017). 